\title{
Evidência de depuração natural da toxina diarréica ácido ocadaico em mexilhões Perna perna (LINNË, 1758) cultivados em fazenda de maricultura na baía de ilha Grande, Angra dos Reis, RJ
}

\author{
Evidence of natural depuration of diarrhetic toxin okadaic acid in \\ mussels Perna perna (LINNĖ, 1758) harvesting in mariculture farm, \\ Ilha Grande, Angra dos Reis, RJ
}

\author{
Aderbson Jorge Lourenço, ${ }^{*}$ Vanessa de Magalhães Ferreira, ${ }^{* *}$ Pedro Paulo de Oliveira da Silva, ${ }^{* * *}$ \\ Carlos A. da R. Rosa, ${ }^{* \star \star *}$ Glória Maria Direito, ${ }^{* * * *}$ Gesilene Mendonça de Oliveira ${ }^{* \star * \star \star *}$
}

\begin{abstract}
Resumo
A toxina diarréica ácido ocadaico $(\mathrm{AO})$ pode ser produzida por algumas espécies de dinoflagelados pertencentes aos gêneros Prorocentrum e Dinophysis. Mexilhões são moluscos filtradores que se alimentam principalmente de fitoplâncton. Quando microalgas toxígenas encontram-se presentes no fitoplâncton os mexilhões podem acumular em sua carne, principalmente na glândula digestiva (hepatopâncreas), ficotoxinas em concentrações suficientes para desencadear no ser humano a síndrome do envenenamento diarréico por moluscos (EDM). A ficotoxina AO é o principal responsável pela síndrome EDM, que é caracterizada por náuseas, dores abdominais, vômitos e diarréia, se forem consumidos moluscos contaminados com

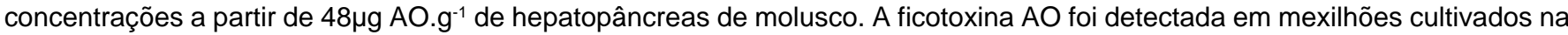
fazenda de maricultura localizada na enseada de Maciéis, baía de llha Grande, RJ. As microalgas foram coletadas com rede de

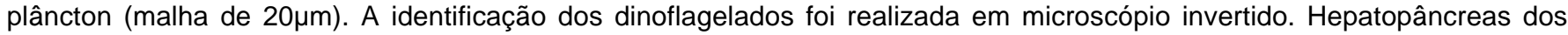
mexilhões coletados foram homogeneizados para extração e detecção de AO. As amostras foram analisadas por Cromatografia Líquida de Alta Eficiência com Detecção Fluorimétrica (CLAE-DF). Os resultados cromatográficos indicaram a presença da toxina $\mathrm{AO}$, em baixa concentração ( 2ng AO.g-1 hepatopâncreas de molusco), em apenas uma das amostras da primeira coleta (março/2004) e sua ausência nas coletas posteriores (abril e maio/2004). Tal fato aponta para ocorrência de depuração natural nos moluscos. O dinoflagelado Dinophysis acuminata é relatado como potencialmente produtor de AO e foi encontrado em densidade muito baixa na área de cultivo, só detectada em amostras concentradas de rede.
\end{abstract}

Palavras-chave: Dinophysis acuminata, mitilicultura, toxina diarréica, depuração.

\begin{abstract}
This work was conducted at the Sea Culture Farm at Maciéis Bay, llha Grande Bay (Angra dos Reis-RJ). During the autumn of 2004, mussels (Perna perna) were collected from the cultivation area and were analyzed to verify the presence of the Phycotoxin okadaic acid (OA). This toxin is produced by dinoflagellates of the genders Prorocentrum and Dinophysis. Mussels that eat these microalgae accumulate the toxin in their flesh, mainly in their digestive glands (hepatopancreas). The ingestion of these mussels by humans can bring about the Diarrhetic Shellfish Poisoning (DSP) syndrome in human being. The toxin (OA) is the main responsible for the DSP syndrome, which is characterized by nausea, stomachache, vomits and diarrhea, if mussels containing over $48 \mu \mathrm{g}$ of OA per gram of hepatopancreas are consumed. The collection of the microalgae was made using a planktonic net (20 $\mu \mathrm{m}$ of mesh). An inverted microscope was used in the identification of the dinoflagellates. The mussels digestive glands were homogenized for the extraction and derivatization of the diarrheic fhycotoxin. The samples were analyzed by High Performance Liquid Chromatography with Fluriometric Detection (HPLC-FD). The chromatographic results indicated the presence of the OA toxin in low concentration ( $\cong 2 n g$ OA per mussel digestive glands grams) in only one of the samples from the first collection (March/2004) and its absence in the following collections (April and May/2004). This demonstrates the occurrence of natural decontamination in the mussels. The dinoflagellate Dinophysis acuminata is a potential producer of the Phycotoxin diarrheic $\mathrm{OA}$ and it was found at very low concentration in the cultivation area, being detected only in concentrated samples collected with the net.
\end{abstract}

Keywords: Dinophysis acuminata, mussels, diarrhetic toxin, depuration.

\footnotetext{
*Biólogo, mestre.

${ }^{* *}$ Oceanógrafa, mestre, professora substituta - Instituto de Biologia, UFRRJ.

***Médico-veterinário, doutor, professor adjunto - Instituto de Tecnologia de Alimentos, UFRRJ.

****Médico-veterinário, doutor, livre-docente - Instituto de Veterinária, UFRRJ.

*****Médica-veterinária, doutora, professora adjunta - Instituto de Veterinária, UFRRJ.

$* * * * * *$ Zootecnista, mestre.
} 


\section{Introdução}

Mexilhões alimentam-se, através de filtração, principalmente do fitoplâncton presente na coluna d'água. O fitoplâncton é uma comunidade constituída por diversos grupos de microalgas, entre elas algumas espécies de dinoflagelados pertencentes aos gêneros Prorocentrum e Dinophysis, potencialmente capazes de produzir toxinas diarréicas implicadas no Envenenamento Diarréico por Moluscos (Yasumoto e Murata, 1993).

$\mathrm{O}$ ácido ocadaico $(\mathrm{AO})$ é a principal toxina diarréica. A presença desta toxina em bivalvos usados na alimentação humana, além de causar distúrbios gastrointestinais, pode promover tumores no sistema digestório humano, quando consumidas regularmente (Daranas et al., 2001). O envenenamento diarréico por moluscos pode ser caracterizado, em evento agudo, com ingestão de doses acima de 48 ug AO (LOAEL) (Van Egmond et al., 1992 apud Vieytes et al., 1997). Os sintomas são náuseas, dores abdominais, vômitos e diarréia. Surgem no intervalo entre 30 minutos até poucas horas após o consumo de molus-cos contaminados. Raramente o quadro clínico manifesta-se passadas mais de 12 horas. Os sintomas cessam após três dias, com ou sem tratamento médico (Yassumoto et al., 1980).

Esta toxina já foi detectada em mexilhões da baía de Sepetiba (Oliveira, 2001; Ferreira, 2004) e em áreas de cultivo no litoral de Santa Catarina (Proença et al., 1998). A microalga suspeita de ter produzido a toxina identificada tanto na baía de Sepetiba quanto no litoral de Santa Catarina foi Dinophysis acuminata. Dinoflagelados pertencentes ao gênero Dinophysis são capazes de produzir toxinas mesmo se presentes em baixa densidade celular na coluna d'água, caracterizando assim a ocorrência de um evento tóxico (Reguera, 2002).

Estudos realizados por Poletti et al. (1996) no mar Adriático demonstraram que mexilhões contaminados naturalmente com uma grande concentração de ácido

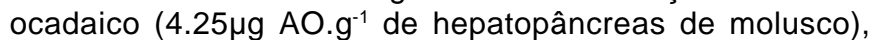
quando transferidos para uma área livre desta toxina, tinham seu nível de contaminação reduzido em $83 \%$ dentro de um período de seis dias. E que após 21 dias a toxina havia desaparecido completamente dos tecidos destes moluscos, configurando-se assim a ocorrência de depuração natural nos mexilhões.

Durante a realização de análises para detectar e quantificar a ficotoxina ácido ocadaico foi observada a evidência da ocorrência de uma possível depuração natural dessa toxina nos mexilhões cultivados na enseada de Maciéis.

\section{Material e métodos}

\section{Área de estudos}

A enseada de Maciéis localiza-se na baía de Ilha Grande, no município de Angra dos Reis, litoral sul do estado do Rio de Janeiro (Figura 1). Juntamente com a baía de Sepetiba funcionam como um sistema estuarino parcialmente misturado (Signorini, 1980).
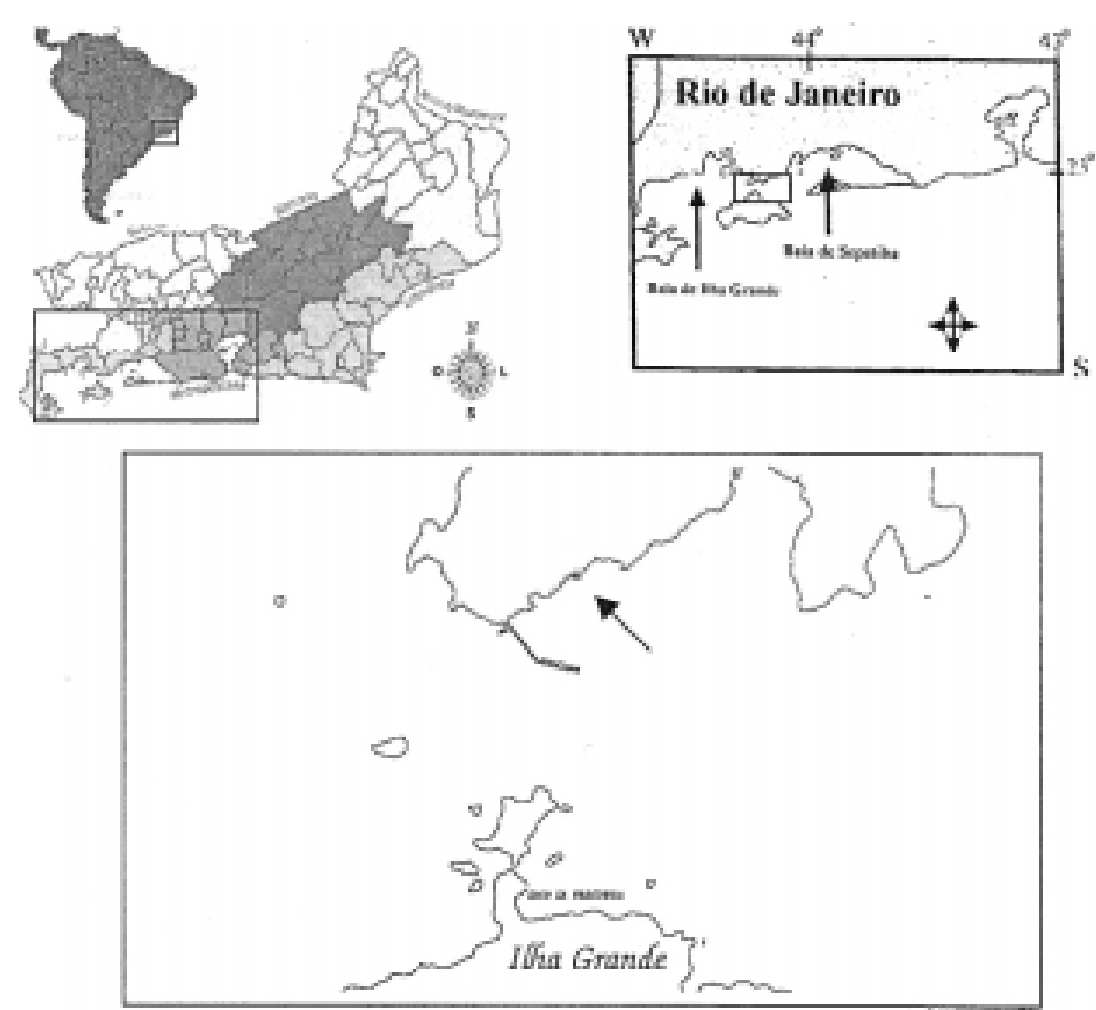

Figura 1: Mapa da baía de Ilha Grande (referência 1) localização Fazenda Marinha Boa Vista de Maciéis - (Coordenada geográfica do centro do cultivo 23 $03^{\prime}$ 03" S / $\left.44^{\circ} 13^{\prime} 33,9^{\prime \prime} \mathrm{W}\right)$

\section{Amostragem (fitoplâncton e mexilhões)}

Segundo Tangen (1978), a distribuição fitoplanctônica ocorre em "manchas", e para coletar e concentrar os organismos, foi utilizada uma rede de plâncton com $20 \mu \mathrm{m}$ de malha. O tipo de arrasto escolhido foi o vertical (integração da coluna d'água), aumentando assim a possibilidade de amostrar microalgas presentes ao longo de toda coluna d'água (Ré, 2001) e também espécies presentes em baixas densidades, como Dinophysis spp. (Reguera, 2002).

Os mexilhões foram coletados em três pontos diferentes da fazenda, na proporção de 50 indivíduos adultos por coleta (para garantir a representatividade estatística), na faixa de tamanho utilizada para comercialização, ou seja, maiores que $6 \mathrm{~cm}$ (Avelar, 1998). 


\section{Identificação do fitoplâncton}

Câmaras de sedimentação, acopladas a cubeta de sedimentação com capacidade para $50 \mathrm{ml}$ de amostra, foram montadas a partir de cada amostra coletada com rede. Após o período de 24 horas, a coluna de sedimentação foi removida, pois as células já haviam decantado no microaquário. Este, coberto com lamínula, foi levado ao microscópio invertido TELAVAL 3 (Carl Zweiss, Germany) para a identificação e quantificação das microalgas. Utilizou-se para a identificação dos dinoflagelados material bibliográfico de Steidinger e Tangen (1997), Taylor et al. (1995) e Balech (2002).

\section{Extração da ficotoxina $A O$}

O ácido ocadaico foi extraído segundo o procedimento descrito por Sigma (1993) e derivado segundo metodologia otimizada por Silva (2001). A ficotoxina AO foi extraída da matriz orgânica do hepatopâncreas dos moluscos com metanol aquoso. Em seguida o extrato metanólico passou por etapas de limpeza que utilizaram éter de petróleo e clorofórmio. Essa última fase foi evaporada a $60^{\circ} \mathrm{C}$ e sob fluxo de nitrogênio. $\mathrm{O}$ resíduo foi redissolvido em acetonitrila.

Como a molécula do $\mathrm{AO}$ não fluoresce naturalmente, para ser detectada necessitou passar pelo processo de derivação onde um cromóforo (1-(bromoacetil)pireno) foi acrescentado na porção carboxílica da molécula da ficotoxina.

\section{Identificação e quantificação do $A O$}

Alíquotas de $20 \mu \mathrm{l}$ da solução derivada foram injetadas no sistema de Cromatografia Líquida de Alta Eficiência (Waters Associats, Inc, Miliford, M.A - USA) equipado com detector de fluorescência Waters (modelo 420) com excitação $333 \mathrm{~nm}$ e emissão 440nm. Utilizou-se injetor Reodyne, com "loop" de $20 \mu \mathrm{l}$. Os dados obtidos foram analisados pelo integrador registrador Waters Data Module (modelo 740). As condições cromatográficas utilizadas foram: fase móvel com acetonitrila e água ultrapura $(85: 15 \mathrm{v} / \mathrm{v})$; fluxo de $1 \mathrm{ml}^{\text {.minuto }}{ }^{-1}$; coluna cromatográfica Microsorb C18 (4,6 X $15 \mathrm{~mm})$ Microsorb-VM ${ }^{\mathrm{TM}}$ $10 \mu \mathrm{m}$ (Varian-USA), a temperatura ambiente.

\section{Resultados e discussão}

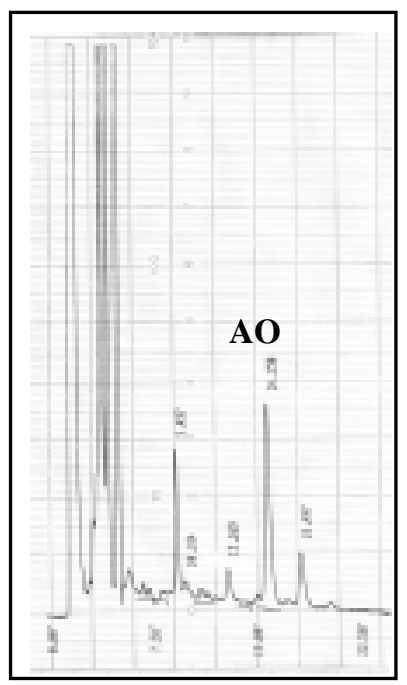

Para que a toxina presente nas amostras pudesse ser identificada, primeiramente foram realizadas injeções com o padrão da toxina ácido ocadaico. Através do tempo de retenção médio (16,524 minutos) encontrado, o pico correspondente ao ácido ocadaico foi identificado (Figura 2). E para posterior cálculo das concentrações da ficotoxina foi utilizada a área média $(88263,80)$ gerada pelo padrão.

Figura 2: Cromatograma do padrão ácido ocadaico com tempo de retenção 16,150 e área de 148434 .
Foram realizadas três coletas no outono de 2004 (março, abril e maio), com três amostragens de mexilhões cada. A ficotoxina ácido ocadaico foi detectada (Figura 3, cromatograma A), com concentração de 2,65 ngAO $\cdot \mathrm{g}^{-1}$ de hepatopâncreas de molusco, em apenas uma amostra das três coletadas no mês de março. Os mexilhões analisados das coletas de abril e maio não apresentaram a presença da ficotoxina ácido ocadáico (Figura 3, cromatogramas B e C).

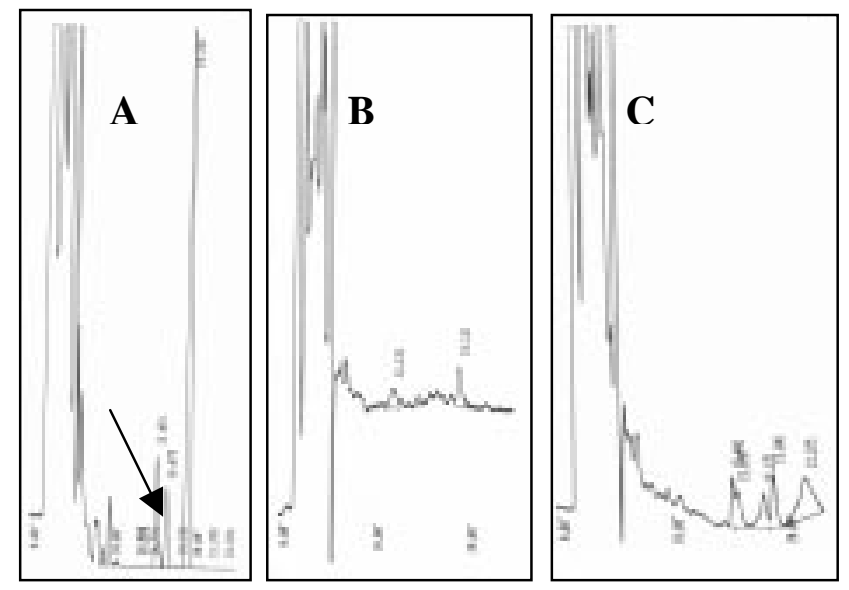

Figura 3: A) Cromatograma da amostra da primeira coleta com a presença da toxina com tempo de retenção de 16,675 e área de 43711; B) Cromatograma da amostra da segunda coleta sem toxina e C) Cromatograma da terceira coleta sem toxina.

O dinoflagelado Dinophysis acuminata (Figura 5) foi identificado nas águas da enseada de Maciéis e foi considerado como suspeito de produzir a ficotoxina $A O$ detectada pelo presente estudo. Proença et al. (1998) implicou a mesma espécie na produção de $A O$, em regiões de cultivo no litoral de Santa Catarina, porém expressando potencial toxígeno uma ordem de grandeza maior (microgramas de AO por grama de hepatopâncreas de molusco) que a encontrada na baía de Ilha Grande.

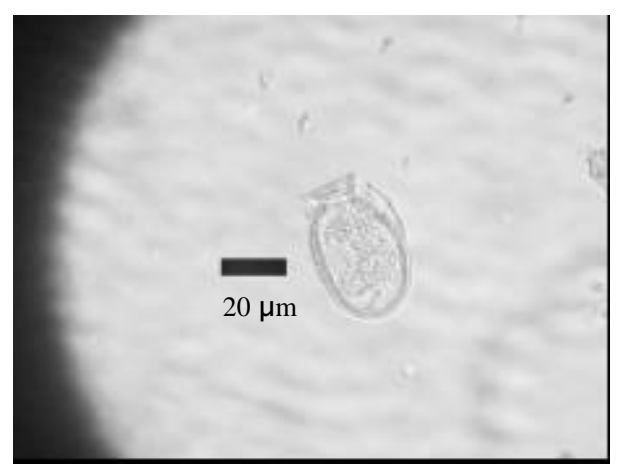

Figura 5: Dinophysis acuminata. Dinoflagelado produtor de ácido ocadaico, coletada na enseada de Maciéis, baía de Ilha Grande (RJ) durante o outono de 2004.

Ferreira (2004) encontrou para mexilhões coletados, em bancos naturais na ilha Guaíba (baía de Sepetiba), a mesma espécie de dinoflagelado produzindo ácido ocadaico ao longo da primavera de 2003 e o verão de 2004, na mesma ordem 
de grandeza que o presente estudo (nanogramas de AO por grama de hepatopâncreas de molusco).

Baseado nesses fatos, sugere-se que $D$. acuminata pode ter produzido a ficotoxina $\mathrm{AO}$ em concentrações maiores, como relatado em Santa Catarina. Tal fato teria ocorrido anteriormente às coletas realizadas pelo presente estudo, pois sabe-se que o AO depura-se naturalmente dentro de um período de 21 dias (Poletti et al., 1996). Então a concentração de AO encontrada no mês de março e sua posterior ausência nos meses de abril e maio configurariam a ocorrência da depuração natural nas águas da enseada de Maciéis.

\section{Referências}

AVELAR, J. C. L. Manual de Mitilicultura. Ministério do Meio Ambiente/Plano Nacional de Meio Ambiente/Plano de Execução Descentralizada. Brasília, DF, 1998. 164 p.

BALECH, E. Dinoflagelados Tecados Tóxicos del Cono Sur Americano In: SAR, E. A., FERRARIO, M. E., REGUERA, B. (Eds.) Floraciones Algales Nocivas en el Cono Sur Americano, Instituto Español de Oceanografía, Vigo, 2002, p.123-144.

DARANAS, A. H., NORTE, M., FERNANDEZ, J. J. Toxic Marine Microalgae. Toxicon, v. 39, 2001. p. 1101-1132.

FERREIRA, V. M. Detecção de ácido ocadaico produzidos por Dinophysis spp. (EHREMBERG, 1839), em mexilhões Perna perna (LINNË, 1858), em situação de primavera e verão, nas ilhas Guaíba e Madeira, baía de Sepetiba, Rio de Janeiro. 2004. 54 f. Tese (Mestrado) - Pós-graduação em Microbiologia Veterinária, Universidade Federal Rural do Rio de Janeiro, 2004.

OLIVEIRA, G. M. Detecção do ácido ocadaico por cromatografia líquida de alta eficiência em mariscos (Perna perna) capturados na baía de Sepetiba. 2001. 85 f. Tese (Mestrado) Instituto de Tecnologia de Alimentos - Universidade Federal Rural do Rio de Janeiro, 2001.

POLETTI, R., VIVIANE, R., CASADEI, C., LUCENTINI, L., GIANNETTI, L., FUNARI, E., DRAISCI R. Decontamination dynamics of mussels naturally contaminated with diarrhetic toxins relocated to a basin of the Adriatic Sea. In: YASUMOTO, T., OSHIMA, Y., FUKUYO, Y. (Eds.). Harmful and toxic Algal Blooms. IOC, UNESCO, 1996. p. 433-436.

PROENÇA, L. A., SCHIMITT, F., COSTA, T., RÖRIG, L. R. Just a diarrhea? Evidence of diarrhetic shellfísh poisoning in Santa Catarina, Brazil. Journal of the Brazilian Association for the Advancement of Science. v. 50, n. 6, November/December 1998, p. 458- 462.

\section{Conclusão}

Pelo fato da toxina ácido ocadaico detectada no mês de março não ter sido mais encontrada nas amostras dos meses de abril e maio, sugere-se que tenha acontecido um processo de depuração natural nos moluscos cultivados nesta enseada. Maiores estudos tanto da composição da comunidade fitoplanctônica quanto dos níveis de toxidez dos moluscos devem ser realizados. Salienta-se, portanto, a urgência na realização de monitoramento de ficotoxinas nas fazendas de maricultura da baía de Ilha Grande para garantir a sanidade dos moluscos e a proteção à saúde pública.

RÉ, P. M. A. B. Capitulo II: Ecologia do Plâncton. In: RÉ, P. M. A. B. Biologia Marinha, Faculdade de Ciências de Lisboa, 2000, p. 19-64.

REGUERA, B. Capítulo 1: Establecimiento de un Programa de Seguimiento de Microalgas Tóxicas. In: SAR, E. A., FERRARIO, M. E., REGUERA, B. (Eds.). Floraciones Algales Nocivas en el Cone Sur Americano. Instituto Español de Oceanografía. 2002. p. 21-54.

SIGNORINI, L.C. A Study of the Circulation in Bay of Illha Grande and of Sepetiba. Part I. A Survey of the Circulation based on Experimental Field Data. Bol. Inst. Oceonogr. S Paulo. Instituto Oceanográfico da Universidade de São Paulo. 1980. p.15-21.

SILVA, P. P. O. Otimização de metodologia para detecção de ácido ocadaico por cromatografia líquida de alta eficiência em moluscos bivalvos. 2001. 62 f. Tese (Doutorado) - Pós-graduação em Ciências Veterinárias - Universidade Federal Rural do Rio de Janeiro. 2001.

SIGMA CHEMICAL CO., St. Louis - USA - 1993.

STEIDINGER, K. A., TANGEN, K. Dinoflagellates In: TOMAS, C. R. (Ed.). Identifying marine phytoplankton. Academic Press, San Diego. 1997. p. 397-583.

TANGEN, K. Nets. In: SOURNIA, A. (Ed.) Phytoplancton Manual. Monographs on Oceanographic Methodology n 6 UNESCO. Paris, 1978, p. 50-58.

TAYLOR, F. J. R., FUKUYO, Y., LARSEN, J. Taxonomy of Harmful Dinoflagellates. In:HALLEGRAEFF, G. M., ANDERSON, D. M., CEMBELLA, A. D. (Eds.) Manual on Harmful Marine Microalgae, IOC Manuals and Guides n. 33, UNESCO. Denmark. 1995, p. 283-317.

VIEYTES, M. R., FONTAL, O. I., LEIRA, F., BAPTISTA DE SOUZA, J. M. V. \& BOTANA, L. M. A Fluorescent microplate assay for diarrhetic shellfish toxins. Analytical Biochemistry. v. 248, 1997. p. 258-264.

YASUMOTO, T., OSHIMA, Y., SUGAWARA, W., FUKUYO, Y., OGURI, H., IGARASHI, T., FUJITA, N. Identification of Dinophysis fortii as the causative organism of diarrhetic shellfish poisoning. Bulletin of the Japanese Society of Scientific Fisheries. v. 46, 1980. p. 1405-1411. YASUMOTO, T., MURATA, M. Marine toxins. Chem. Rev. v. 93, 1993, p. 1897-1909. 